\title{
Continuous Glucose Monitoring with Multiple Daily Insulin Treatment: Outcome Studies
}

\author{
Janet B. McGill, MD ${ }^{1}$ and Andrew Ahmann, $M D^{2}$
}

\begin{abstract}
Continuous glucose monitoring (CGM) is developing into an increasingly useful tool for glucose monitoring and therapeutic guidance in the treatment of diabetes. Multiple daily doses of insulin (MDI) is the most common method for intensive insulin treatment of type 1 diabetes and is also becoming more common in the treatment of type 2 diabetes as an increasing population with type 2 diabetes experiences progressive beta cell loss. The clinical evidence demonstrating the benefit of CGM in the outcomes of patients treated with MDI is becoming clearer, particularly with the recent completion of several randomized clinical trials addressing both type 1 and 2 diabetes. This evidence is reviewed.
\end{abstract}

Keywords: Continuous glucose monitoring, Multiple daily doses of insulin, Type 2 diabetes, Type 1 diabetes, Hypoglycemia, Diamond Study.

\section{Introduction}

T HAS BEEN over 20 years since the association between glucose control and diabetes complications became irrefutable. ${ }^{1-3}$ During this interval there has been great focus on development of pharmacological agents, including improved insulin products and multiple noninsulin agents with various mechanisms of action. ${ }^{4,5}$ Yet, many individuals with diabetes fail to meet glucose goals considered appropriate for the prevention of chronic complications. ${ }^{6-8}$ Patients are faced with numerous barriers to improved control, including inconvenience, cost, inertia in changing personal health habits, fear of weight gain, and fear of hypoglycemia.

Since the publication of the DCCT in $1993,{ }^{1}$ insulin regimens have become increasingly complex in the effort to make the treatment of type 1 diabetes more effective, physiologic, and flexible. At the same time, it has become obvious that improved guidance for insulin dosing is necessary to attain lower glucose averages and improved consistency while avoiding hypoglycemia, an acute complication of insulin therapy that can be life threatening. Self-monitoring of blood glucose (SMBG) with personal meters became a practical companion to insulin treatment starting in the 1980s and was a necessary tool to conduct the DCCT trial of intensified insulin therapy. Over the years, technology for SMBG became more convenient and more accurate. The evidence indicates a direct association between frequency of SMBG and glucose control in type 1 diabetes with lowest HbA1c seen in those monitoring at least 10 times daily. ${ }^{9}$ Many patients find it difficult to practice SMBG monitoring at recommended rates despite the recognized benefit.

The introduction of continuous glucose monitors to clinical medicine in 2000 emanated from the belief that attainment of frequent glucose levels with less frequent effort by the patient would lead to improved guidance and improved outcomes. ${ }^{10,11}$ Again, over the intervening years the CGM devices have become more accurate with improved analytical tools and improved patient usability. In their 2017 standards of care, the American Diabetes Association (ADA) states that "when used properly, CGM in conjunction with intensive insulin regimens is a useful tool to lower A1C" citing the highest level evidence. ${ }^{12}$ Likewise, the Endocrine Society and the American Association of Clinical Endocrinologists support CGM use in type 1 diabetes. ${ }^{13,14}$ However, as noted

\footnotetext{
${ }^{1}$ Washington University in St. Louis, St Louis, Missouri.

${ }^{2}$ Oregon Health \& Science University, Portland, Oregon.
} 
by Yeh et al. in a systematic review, the available evidence is essentially restricted to the use of CGM with insulin pumps. ${ }^{15}$ The majority of individuals with type 1 diabetes in the United States are treated with multiple daily doses of insulin (MDI) rather than with continuous subcutaneous insulin infusions (CSII). Data on the combined use of CGM with MDI have clearly been insufficient. For this and other reasons the use of MDI continues to be infrequently utilized in clinical practice despite the technological advancements. It is likely that the $10 \%$ CGM use reported among the adult patients in the recent T1D (type 1 diabetes) Exchange Report represents a bestcase scenario as this is a select group of patients from progressive diabetes centers, including an unusually high $60 \%$ on insulin pump therapy. ${ }^{6}$ Beyond the issue of evidence for benefits of CGM in individuals with type 1 diabetes on MDI is the question of potential benefit for those patients with type 2 diabetes. Increasing number of patients with type 2 diabetes has progressed to intensive insulin therapy and the benefit of CGM in this treatment group has not been appropriately addressed. We are now beginning to see progress in clinical research addressing the use of CGM in those treated with MDI, in both type 1 and 2 diabetes.

\section{CGM in Type 1 Diabetes Treated with MDI}

Treatment challenges, morbidity, and mortality in type 1 diabetes

Insulin replacement therapy in type 1 diabetes continues to be problematic despite advancements in insulin formulations, in insulin delivery systems, and in methods of SMBG. In a follow-up study of 16,061 persons enrolled in the T1D (type 1 diabetes) Exchange Clinic Registry, the mean HbA1c increased slightly from $8.2 \%$ to $8.4 \%$ from the initial survey in 2010-2012 to the follow-up in 2013-2014. ${ }^{6}$ Lower mean HbA1c was noted in adults compared with children, and in those using an insulin pump and/or CGM compared with those using MDI. ${ }^{6}$ SMBG was performed, a mean of 4.7 times per day, range 3.5 to 6.2 times per day in the follow-up cohort. Investigators for the T1D Exchange Clinic Registry had previously demonstrated that the frequency of SMBG was inversely related to $\mathrm{HbA1c}$ in all age groups, and that frequent monitoring $(6.45 \pm 2.94$ times/day vs. $4.24 \pm 2.30$ times/day, $P<0.0001)$ was a key factor in the achievement of excellent glucose control, defined as HbA1c $<6.5 \%$ versus those in fair to poor control, HbA1c $\geq 8.5 \%{ }^{9,16}$ Other characteristics such as higher socioeconomic status and older age, and behaviors such as exercising frequently, giving mealtime bolus doses before eating, and missing bolus doses less frequently were also more common in the excellent control group compared with the fair/poor control group. ${ }^{16}$

Attainment of the ADA and EASD (European Association for the Study of Diabetes) HbA1c goal of HbA1c $<7 \%$ is challenging for the majority of patients with type 1 diabetes. In addition to well-described chronic complications, higher HbA1c is associated with increased mortality in both the DCCT-EDIC study group and in a Swedish population-based study of persons with type 1 diabetes compared with controls. ${ }^{17,18}$ In the DCCT-EDIC mortality study, intensive insulin therapy nearly obliterated the difference in cardiovascular outcomes at 30 years of follow-up, but in the full cohort, a $10 \%$ higher $\mathrm{HbA1c}$ was associated with a $56 \%$ increase in the risk of mortality. ${ }^{17,19,20}$ Reports from Scotland, Australia, and
Norway show that both acute and chronic complications of diabetes contribute to the excess mortality in persons with type 1 diabetes. ${ }^{21-23}$ Sudden death, including those discovered dead-in-bed accounted for $22 \%$ of the deaths in persons dying under age 40 with type 1 diabetes in the Australian study and $5 \%$ of those dying before age 30 in the Norwegian cohort. ${ }^{21,24}$ One case report confirmed hypoglycemia as the cause of deadin-bed syndrome, and a recent report by Novodvorsky et al. confirms that cardiac arrhythmias and repolarization occur during asymptomatic nocturnal hypoglycemia more commonly than daytime hypoglycemia.

Hypoglycemia is a well-described deterrent to the achievement of normal glycemia and impacts patients at all levels of glucose control. Mild hypoglycemia is common in patients with type 1 diabetes, occurring daily to weekly. ${ }^{25}$ Even when self-treatment is possible, hypoglycemic episodes disrupt daily life, impair cognitive function and performance, and can result in injury. ${ }^{28}$ Severe hypoglycemia that requires the assistance of another person, or is sometimes defined as a blood glucose of $\leq 54 \mathrm{mg} / \mathrm{dL}$ ( $3 \mathrm{mmol} / \mathrm{L})$, can result in seizure or coma in $25 \%$ of cases and death very rarely. ${ }^{28}$ Frequent episodes of hypoglycemia may lead to impaired awareness of hypoglycemia (IAH). Education aimed at physiologic dosing of insulin and recognition of activities that may lead to hypoglycemia, and continuous infusion of insulin through insulin pumps with personalized settings have provided some benefit to patients with IAH, but do not abolish the risk of severe hypoglycemia entirely. ${ }^{29}$ In a review and metaanalysis of CSII versus MDI for the reduction of severe hypoglycemia, Pickup and Sutton found that CSII reduced severe hypoglycemia and reduced HbA1c by $0.62 \%$, but in most of the studies cited patients had high starting HbAlc levels and rarely got to goal. ${ }^{30}$

\section{Initial studies of CGM utility in type 1 diabetes using MDI}

CGM has changed the conversation in type 1 diabetes. No longer do intervention studies consider only $\mathrm{HbA} 1 \mathrm{c}$ and frequency of hypoglycemia, but now routinely report time spent in range $(70-180 \mathrm{mg} / \mathrm{dL})$, above range $(>180 \mathrm{mg} / \mathrm{dL})$, and below range (usually $<70 \mathrm{mg} / \mathrm{dL}$ ) as measured with either real-time or blinded CGM. Lowering of HbA1c remains an important target of therapeutic interventions, but not at the expense of increased time in the hypoglycemic range, or with increased frequency of hypoglycemia. Improved accuracy of CGM systems has permitted these changes.

The effectiveness of CGM in patients with type 1 diabetes using MDI has been examined in nonrandomized observational and intervention studies, and in randomized clinical trials (Table 1). In the very first report of real-time CGM use in the outpatient setting, Garg et al. showed that CGM users ( 7 of 15 were on MDI, 8 of 15 on CSII) spent less time below $3.1 \mathrm{mmol} / \mathrm{L}(P<0.05)$ and $25 \%$ less time above $13.3 \mathrm{mmol} / \mathrm{L}$ $(P<0.05)$ during the unblinded portion of the study compared with the blinded period. ${ }^{31}$ A 12 -week trial of the STS $^{\circledR}$ System (DexCom, Inc., San Diego, CA) in 139 patients, of whom 109 had type 1 diabetes and 57 were treated with MDI, showed a HbA1c reduction of $0.4 \%, P<0.001$, in the MDI subgroup. ${ }^{32}$ The first and largest randomized study of CGM use in children and adults was funded by the Juvenile Diabetes Research Foundation. ${ }^{33}$ Of the 322 participants, 20\% 


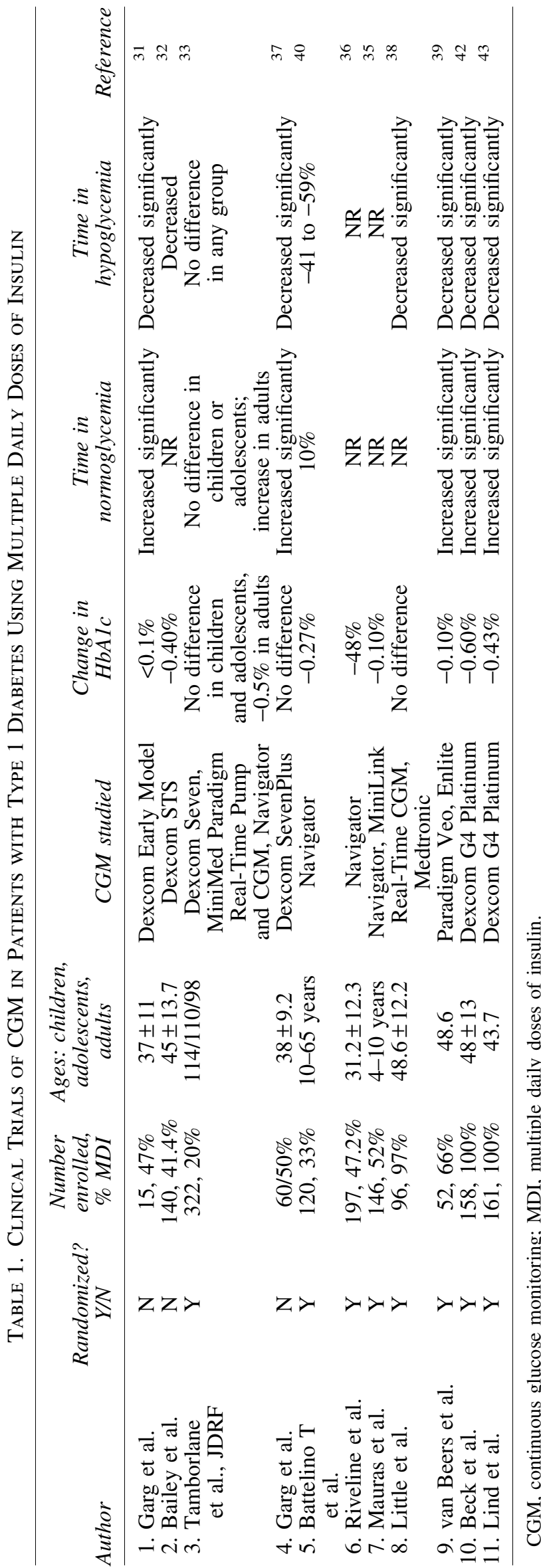

used MDI, with the greatest percentage of MDI users in the adolescent cohort. The results showed that adults age $\geq 25$ years experienced a reduction in $\mathrm{HbA} 1 \mathrm{c}$ of $0.53 \%(95 \%$ confidence interval $[\mathrm{CI}]-0.71$ to $-0.35 ; P<0.001)$, but there was no difference in children or adolescents. ${ }^{33}$ In the $12-$ month extension of the same study, adults with a baseline HbA1c of $>7 \%$ experienced a mean change in HbA1c of $-0.4 \% \pm 0.6 \%(P<0.001)$, reduced incidence of severe hypoglycemia and increased time-in-range of $71-180 \mathrm{mg} / \mathrm{dL}$ from baseline to 12 months. ${ }^{34}$ The participants used either a DexCom SEVEN (DexCom), MiniMed Paradigm REALTime System (Medtronic MiniMed, Northridge, CA) or FreeStyle Navigator (Abbott Diabetes Care, Alameda, CA) system and data were not presented per type of insulin administration or sensor type. ${ }^{33,34}$

A follow-up study by The Diabetes Research in Children Network (DIRECNET) Study Group reported by Mauras et al. tested CGM in 146 children with type 1 diabetes. ${ }^{35}$ In this 26 -week study, $36 \%$ of the participants used MDI. There was no change in HbAlc or hypoglycemia in either the CGM or control groups. CGM use declined over the 26 weeks of the study, however. A key factor in the success of CGM to lower HbAlc and to reduce hypoglycemia is clearly wear time and use of trend arrows and alarms to help guide therapy and to provide warning for hyperglycemia or impending hypoglycemia. ${ }^{35}$

Riveline et al. reported a three-arm study comparing standard of care versus physician- or patient-led groups using CGM. $^{36}$ The study population from 19 diabetes care centers in Europe included 178 adults, mean age 36.4 13.6 years, diabetes duration about 17 years, $47.2 \%$ using MDI, mean HbA1c $9.0 \% \pm 0.9 \%$. The Freestyle Navigator CGM (Abbott Diabetes Care) was used for the study. Interestingly, over the 1-year duration of the study, the HbA1c dropped more in the patient-led group $(-0.50 \%, 95 \% \mathrm{CI}-0.70 \%$ to $-0.29 \%)$ and the physician-led group $(-0.45 \%, 95 \% \mathrm{CI}-0.66 \%$ to $-0.24 \%)$ than in the standard treatment group $(0.02 \%, 95 \% \mathrm{CI}-0.18$ to 0.23$){ }^{36}$ The combined CGM groups had superior HbAlc reduction compared with standard treatment, $-0.48 \%(95 \%$ CI -0.63 to -0.33$), P<0.0001$; however, the CSII-treated patients had a more robust drop than MDI-treated patients, $-0.67 \%$ compared with $-0.28 \%, P=N_{S}{ }^{36}$ Sensor use was 57 to $65 \pm 20-29 \%$ of the prescribed time, with the patient-led group utilizing the sensor for a greater percent of time than the physician-led group. Hypoglycemia was not different between the CGM and standard treatment groups. ${ }^{36}$

Garg et al. performed a pilot study to examine the relative glucose changes seen in type 1 patients adding CGM to therapy with MDI or CSII. ${ }^{37}$ The study suffered from a high dropout rate over the 6 months and found little change in A1C. However, the findings were similar for the MDI- or CSII-treated patients.

\section{Impact of CGM on hypoglycemia and hypoglycemia unawareness}

The problem of hypoglycemia unawareness affects up to $20 \%$ of adults with long-standing type 1 diabetes, and interferes with their ability to achieve excellent glucose control. Two studies have addressed the utility of CGM specifically in this group of patients. Little et al. reported the results of a $2 \times 2$ factorial randomized clinical trial adults with type 1 diabetes and IAH, testing whether CSII versus MDI and CGM 
versus SMBG would improve hypoglycemia awareness as measured by the Gold criteria. ${ }^{38}$ At the end of the 24 -week treatment period, the frequency of hypoglycemia $(\leq 3 \mathrm{mmol} / \mathrm{L})$ and hypoglycemic awareness improved without a change in $\mathrm{HbA1c}$. No differences were detected in any of the outcomes between CSII and MDI or between SMBG and CGM. ${ }^{38} \mathrm{~A}$ similar group of patients with IAH and at high risk for severe hypoglycemic events were enrolled in the study, "Continuous Glucose Monitoring for Patients with Type 1 Diabetes and Impaired Awareness of Hypoglycemia (IN CONTROL)," 39 Of the 52 subjects, 29 used MDI and 23 used CSII for insulin administration. IN CONTROL employed a crossover trial design, where subjects were randomized to 16 weeks of CGM use followed by 12 weeks of washout and 16 weeks of SMBG with blinded CGM or the reverse sequence. ${ }^{39}$ The results of IN CONTROL showed that while using CGM, subjects spent more time in normoglycemia: $65.0 \%$ (95\% CI 62.8-67.3) versus $55.4 \%$ (53.1-57.7; difference 9.6\%, CI 8.0-11.2; $P<0.0001$ ); and reductions in mild and moderate hypoglycemia were noted. Additionally, the number of severe hypoglycemic events that occurred during the trial was reduced (14 vs. 34 events, $P=0.033) .{ }^{39}$ Results were not reported separately for MDI versus CSII, except a notation that neither the sequence of the treatment allocation nor the type of insulin administration altered the findings.

Reduction in hypoglycemia was the specified outcome of a randomized, controlled clinical trial in children and adults whose $\mathrm{HbA} 1 \mathrm{c}$ was $<7.5 \%$ at screening. ${ }^{40}$ Of the 120 patients, $33 \%$ used MDI and $44 \%$ were children. After the 26-week intervention period, a greater reduction in time spent in hypoglycemia was demonstrated for the CGM group versus the SMBG group $(0.48 \pm 0.57 \mathrm{~h} /$ day vs. $0.97 \pm 1.55 \mathrm{~h} /$ day respectively). ${ }^{40} \mathrm{HbA} 1 \mathrm{c}$ was also lower in the CGM group than the control group (difference $-0.27 \%$, 95\% CI -0.47 to $-0.07 ; P=0.008) .{ }^{40}$ Time spent in the normoglycemic range was increased in the CGM group compared with controls.

\section{Recent studies of CGM in MDI-treated patients with type 1 diabetes}

The adoption of CGM technology has been greater among persons with type 1 diabetes using CSII than MDI, as reflected in an observational survey from the T1D Exchange Clinic Registry. ${ }^{41}$ Of a total of 17,731 participants with duration of type 1 diabetes $>1$ year, only 2\% used CGM with MDI, whereas $13 \%$ used CGM in addition to CSII. ${ }^{41}$ Among CGM users, the mean HbA1c was not different between CSII and MDI users, and was lower than non-CGM users $(7.6 \%$ and $7.7 \%$ in CSII and MDI, respectively vs. $8.3 \%$ and $8.8 \%$ in CSII and MDI cohorts, respectively, $P<0.001$ for both comparisons). In adults $\geq 26$ years, the mean $\mathrm{HbA1c}$ in the CSII plus CGM and MDI plus CGM was identical at $7.3 \%$, suggesting that CGM obliterated the glucose control advantage typically seen with CSII. ${ }^{41}$

Two studies published in 2017 have addressed the questions of overall effectiveness of CGM in improving glycemic control in type 1 diabetes patients using MDI, including lowering of HbA1c, time in range, and time spent in hypoglycemia. The DIAMOND (Effect of Continuous Glucose Monitoring on Glycemic Control in Adults with Type 1 Diabetes Using Insulin Injections) and GOLD (Continuous Glucose Monitoring vs. Conventional Therapy for Glycemic
Control in Adults with Type 1 Diabetes Treated with Multiple Daily Injections) randomized clinical trials specifically targeted MDI-treated patients to evaluate the added benefit of CGM in addition to SMBG versus SMBG alone. ${ }^{42,43}$ Both studies used the DexCom G4 Platinum CGM system. In DIAMOND, 152 participants, mean age 48, mean baseline HbA1c $8.6 \%$, were randomized to CGM plus SMBG or SMBG alone for 24 weeks. The study had a high completion rate of $98 \%$, and mean wear time of $>6$ days in $93 \%$ of participants at the end of the 6-month trial period. The mean HbA1c dropped in both groups to $-1.0 \%$ in the CGM group and $-0.4 \%$ in the SMBG group for an adjusted treatment-group difference of $-0.6 \%(95 \% \mathrm{CI}-0.8$ to $-0.3 ; P<0.001))^{42}$ The time spent in hypoglycemia $(<70 \mathrm{mg} / \mathrm{dL}$ ) was $43 \mathrm{~min} /$ day versus $80 \mathrm{~min} /$ day in the CGM versus SMBG groups, respectively, $P=0.002$. $^{42}$ The GOLD trial used a crossover design with half of the 161 randomized participants using CGM for 6 months in addition to SMBG, followed by a 4-month washout period, then 6 months of usual care with SMBG alone, whereas the other half of the participants performed SMBG for the initial 6 months, and washout period followed by 6 months of CGM. ${ }^{43}$ Followup data were available for 142 participants, who wore the CGM between $86.5 \%$ and $91.9 \%$ of the time assigned. From a baseline $\mathrm{HbA} 1 \mathrm{c}$ of $8.6 \%$, the mean HbA1c during CGM use was $7.92 \%$, and during conventional treatment was $8.35 \%$, mean difference $-0.43 \%(95 \% \mathrm{CI}-0.57 \%$ to $-0.29 \%$, $P<0.001){ }^{43}$ Time spent in hypoglycemia was $2.79 \%$ during the CGM period and $4.79 \%$ during conventional therapy. ${ }^{43}$ Notably, significant improvement was noted in subjective wellbeing and treatment satisfaction, and hypoglycemia confidence improved during the CGM treatment period in GOLD. ${ }^{43}$ The conclusions drawn from these studies were summarized by Davidson, who stated, “...the clinical trials by Beck et al. and by Lind et al. involving patients with type 1 diabetes who receive insulin via multiple daily injections demonstrate that compared with SMBG, CGM limits hyperglycemia and hypoglycemia, improves diabetes control, and reduces glucose variability." 44

As the prevalence of type 1 diabetes increases, clinicians and healthcare systems will be challenged to find better management strategies that are patient centric, and that reduce both the acute and chronic risks of the disease. ${ }^{45}$ CGM has become an invaluable tool for patients with type 1 diabetes, whether they are using CSII or MDI. HbA1c improvements in the long-term studies have ranged between $0.3 \%$ and $0.6 \%$, and hypoglycemia is generally, but not always reduced when CGM is compared with SMBG (Table 1). An economic analysis reported by Bronstone and Graham found that the reduction in severe hypoglycemia requiring medical assistance from use of CGM would save between $\$ 936$ and $\$ 1346$ per person annually. ${ }^{46}$ Reductions in the costs of long-term complications and lives saved are also likely, depending on availability of the technology and implementation across the population of patients with type 1 diabetes.

\section{CGM in Type 2 Diabetes Treated with MDI}

\section{Evidence for general benefit of CGM in type 2 diabetes}

There is good reason to believe that CGM would be a useful tool for those with type 2 diabetes. CGM is an excellent teaching tool for patients as well as a guide for medical professionals to direct changes in activity and dietary habits 
and highlight the benefits of medication adherence. Use of short-term, intermittent retrospective CGM has demonstrated benefit in type 2 diabetes in noninsulin-treated patients. For example, in a small randomized study of noninsulin-treated patients, Allen et al. reported significantly improved HbAlc with a behavioral approach that resulted in weight loss and increased physical activity. ${ }^{47}$ Yoo et al. studied 65 patients with poorly controlled T2D on a variety of treatments. ${ }^{48}$ The focus of the CGM was on education and promotion of lifestyle changes and resulted in a $0.7 \%$ greater $\mathrm{HbA} 1 \mathrm{c}$ reduction in the CGM intervention group than in the group randomized to SMBG alone with associated changes in weight, exercise, and postprandial glucose. ${ }^{49}$

The accepted value of blinded CGM is supported by the increasingly frequent use of blinded CGM to compare glucose responses to a wide variety of pharmacological agents in type 2 diabetes. ${ }^{49-55}$ Such studies have been able to characterize treatments, including direct comparison of agents with regard to postprandial effects, glucose mean, hypoglycemic exposure, and overall variability. However, the demonstration of the CGM clinical impact in type 2 diabetes is marred by reports that fail to allow for clear conclusions. Most of the studies are small, include mixed therapeutic approaches in the intervention group, and are seldom prospective and randomized. ${ }^{56}$ Many studies have used intermittent blinded CGM, although there has been increasing emphasis on realtime or personal CGM. No large studies have been performed exclusively in noninsulin-treated type 2 diabetes and randomized trials have been infrequently reported in this group.

\section{Potential benefit of CGM in type 2 patients on insulin: risk of hypoglycemia}

More pertinent to this discussion, CGM would be expected to show benefits in those patients with type 2 diabetes treated with insulin and particularly in patients on MDI. Beyond the behavioral benefits possible in those receiving no pharmacological therapy or receiving noninsulin agents, CGM has more specific potential in those treated with MDI as shown in the patients on MDI with type 1 diabetes, where HbA1c is lowered and the hypoglycemic exposure is decreased. The rate of hypoglycemia in type 2 diabetes is higher than usually appreciated. $^{57-59}$ For example Lipska et al. reported that $10.8 \%$ of patients in the large database experienced severe hypoglycemia. ${ }^{59}$ Gehlaut et al. found that $49 \%$ of patients with type 2 diabetes in their study had at least one episode of hypoglycemia during a 5-day analysis with CGM and many of those hypoglycemic episodes were asymptomatic. ${ }^{58}$ They also found that $21 \%$ of those patients found to have hypoglycemia had values $<50 \mathrm{mg} / \mathrm{dL}$ and episodes were most common in those on insulin. Cariou et al. ${ }^{57}$ evaluated a mixed population of patients with type 1 and 2 diabetes in an observational study over 30 days and found that $45 \%$ of patients reported at least one episode of hypoglycemia. Using two or more injections of insulin per day was a predictor of increased hypoglycemia. ${ }^{57}$ Finally, Pazos-Couselo et al. evaluated 63 insulin-treated patients with type 2 diabetes and showed that CGM over 1 week identified more hypoglycemia than was found on 8 weeks of monitoring with SMBG. ${ }^{60}$ Fifty-nine percent of the patients had hypoglycemia by CGM and $30 \%$ had nocturnal hypoglycemia. Likewise, the UKPDS data confirmed the increased frequency of hypoglycemia among those with type 2 diabetes who were treated with MDI relative to basal insulin or noninsulin therapy. ${ }^{61}$ Although the frequency of hypoglycemia is lower in type 2 diabetes than in type 1 diabetes, the consequences may be greater as this older population with higher cardiovascular risk may be particularly affected. For example, there has been reported $>2.5$-fold increase in cardiovascular death and in major cardiovascular events in those with type 2 diabetes in the ADVANCE Trial who experienced severe hypoglycemia. ${ }^{62}$ Increased arrhythmias have been shown with hypoglycemia and multiple mechanisms have been proposed for hypoglycemia-induced cardiovascular events, including prolonged QT. ${ }^{62-65}$ Therefore, for patients with type 2 diabetes treated with intensive insulin therapy, tools to reduce hypoglycemia could be critically important.

\section{Evidence of broad benefits of CGM in type 2 patients on insulin}

Additionally, reports of CGM benefits in evaluating postprandial patterns, related meal insulin dosing, including dynamics of rapid-acting insulin, adequacy of basal insulin when not eating, correction responses, patterns of hypoglycemia, and impact of exercise or other lifestyle variables expand the expectations of CGM in assisting with management of type 2 diabetes patients on intensive insulin therapy. ${ }^{66,67}$ Unfortunately, studies addressing CGM use in type 2 patients treated with insulin have yielded very limited information. Many studies have focused on patients with varied therapies of type 2 diabetes mellitus rather than focusing on those specifically using MDI, whereas other studies included insulin-treated patients with type 1 and 2 diabetes. Ehrhardt et al. studied 100 patients with type 2 diabetes using realtime CGM for 2 of every 3 weeks over a 12 -week period in a randomized controlled trial. ${ }^{68}$ The authors reported a $0.5 \%$ greater $\mathrm{HbA} 1 \mathrm{c}$ reduction in the patients using CGM. However, the study does not pertain to our discussion of MDI therapy because while 19 of the 50 patients in the CGM group were on basal insulin, prandial insulin was a study exclusion. At the 12-week study point, these investigators reported significant heterogeneity of response with CGM. ${ }^{69}$ The same group extended this study to 52 weeks, where a final $0.6 \% \mathrm{HbA} 1 \mathrm{c}$ difference was demonstrated. ${ }^{70}$

\section{Earlier studies of CGM in type 2 diabetes treated with MDI}

Finally, data on the results of CGM in type 2 patients treated with MDI is not easily extracted. The report from Garg et al. in 2006 included 91 insulin-treated patients. ${ }^{71}$ In this 9-day trial, the researchers demonstrated decreased time in the hypoglycemic range and hyperglycemic range and $26 \%$ increased time in the target range with real-time CGM. However, they did not separate out the results in the 16 patients who had type 2 diabetes. Bailey et al. completed a 12-week observational trial in 140 adults with type 1 or type 2 diabetes. ${ }^{32}$ Among the 57 total patients on MDI (23 with type $2 \mathrm{DM}$ ) the reduction in $\mathrm{HbA} 1 \mathrm{c}$ was $0.5 \%$ over the 12 weeks, but the results in type 2 patients on MDI were not reported separately. Interestingly, the type 1 patients on CSII had less $\mathrm{HbA} 1 \mathrm{c}$ reduction at $0.3 \%$. Cosson et al. reported the first randomized trial of CGM use in insulin-treated patients, but used only $48 \mathrm{~h}$ of glucose data to alter therapy and the study 
only included three patients with type 2 diabetes on insulin. ${ }^{72}$ Therefore, little can be gained from this study. Another randomized trial of insulin-treated patients found significant differences in time out of range and reduced hypoglycemia in those using CGM with or without alarms. ${ }^{73}$ However, the study was a mixed population of patients with type 1 and 2 diabetes and there were only six patients with type $2 \mathrm{DM}$ in each group. The HbA1C levels were not significantly different after this 3-month study, although twice as many patients experienced an $\mathrm{HbA} 1 \mathrm{c}$ reduction of at least $0.5 \%$ (NS).

Up until the recently reported data from the DIAMOND trial, the best information relevant to the discussion of CGM in MDI-treated type 2 patients comes from somewhat unique populations. Murphy et al. showed several benefits of CGM in patients with type 1 or 2 type diabetes during pregnancy. ${ }^{74} \mathrm{~A}$ pilot study evaluated the benefit of CGM in type 2 patients with ESRD receiving hemodialysis and being treated with MDI. ${ }^{75}$ In this study, there was a decreased HbA1c, mean CGM glucose, and hyperglycemia (over $180 \mathrm{mg} / \mathrm{dL}$ ) with the CGM intervention. The study did not see a change in hypoglycemia.
In a randomized trial using flash glucose sensing as a replacement for SMBG, Haak et al. found no difference in HbA1c after 6 months, but did find nearly 30 min less time daily in the hypoglycemic range in those on the sensor. ${ }^{76}$ However, flash glucose sensing differs from CGM in frequency of results, automated trending information, and the use of alarms.

\section{Prospective RCT of real-time CGM in type 2 diabetes treated with MDI}

In summary, data on CGM use in patients with type 2 DM treated with MDI are fragmented. The better studies suggest an $\mathrm{A} 1 \mathrm{C}$ reduction of about $0.5 \%-0.6 \%$ when CGM is applied in this population, but there have been no studies that were randomized controlled trials addressing the specific question until the recent presentation in abstract form of the type 2 component of the DIAMOND trial. ${ }^{77}$ In this study, 158 patients with type 2 diabetes treated with a basal-bolus insulin regimen were randomized to use of the Dexcom G4 CGM

\section{a Primary Outcome: Mean Reduction in A1C with CGM vs Usual Care}

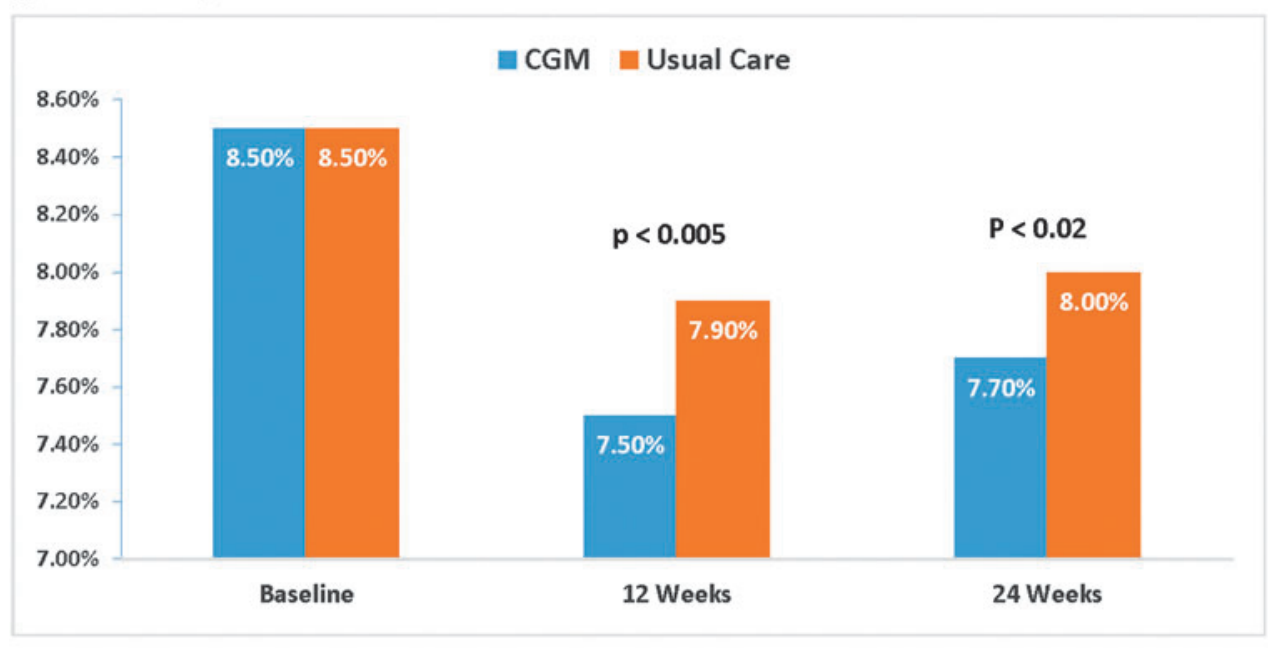

b Secondary A1C Outcomes

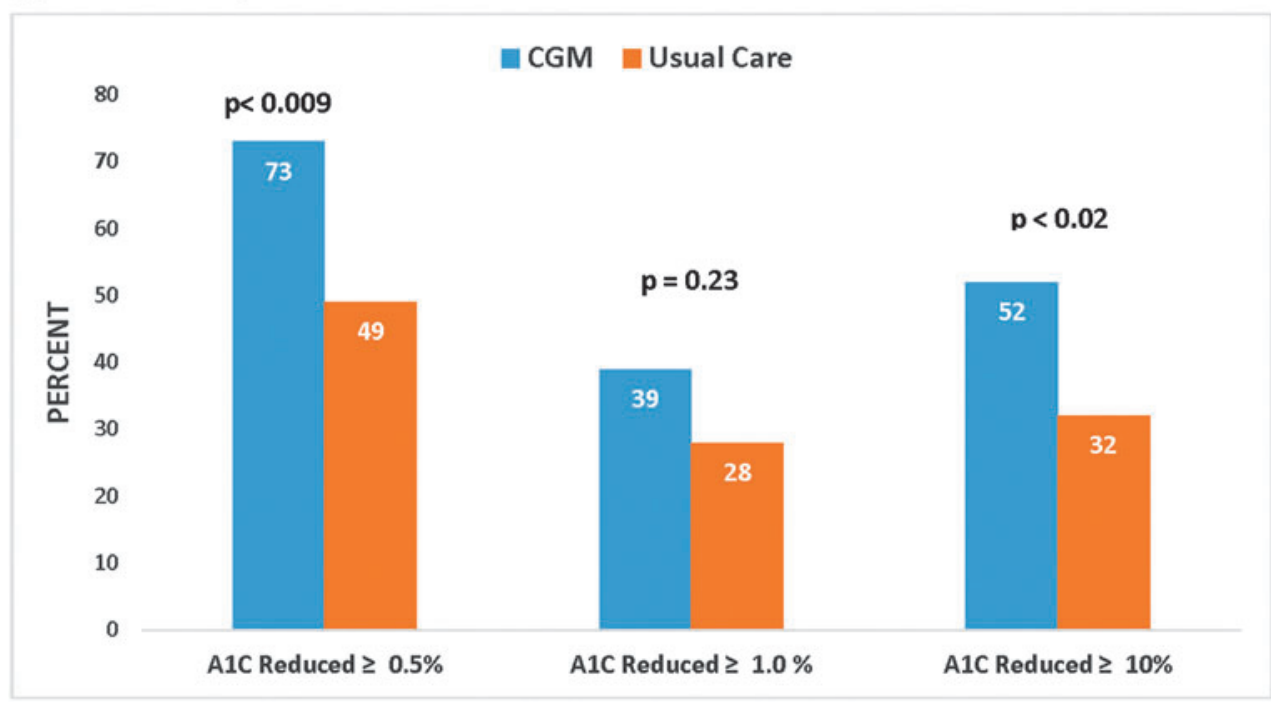

FIG. 1. HbA1c outcomes in type 2 DIAMOND trial. Adapted from Bergenstal et al. ${ }^{77}$ with permission. 


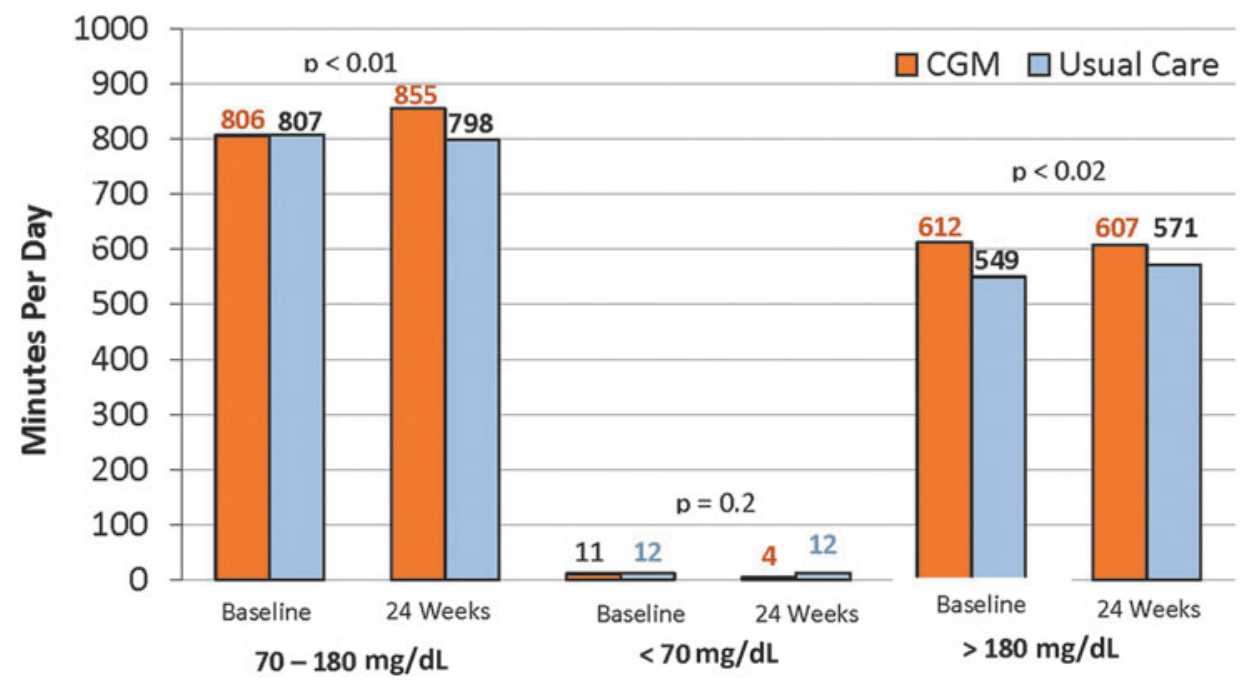

FIG. 2. CGM metrics as secondary outcome in type 2 DIAMOND trial. Time in range (70-180 mg/dL), time in hypoglycemia $(\leq 70 \mathrm{mg} / \mathrm{dL})$, and time in hyperglycemia $(\geq 180 \mathrm{mg} / \mathrm{dL})$. Adapted from Bergenstal et al. ${ }^{77}$ with permission.

with 505 software or SMBG for treatment guidance. As with the type 1 component of the study discussed above, ${ }^{42}$ the emphasis was on a practical approach minimizing patient contact between relatively conservatively spaced clinic visits, including no visits or contact between the 12- and 24 -week visits. The reported results included a reduction in HbA1c of $1.0 \%$ in the CGM-treated group at 12 weeks and $0.8 \%$ reduction at 24 weeks. However, the control group (SMBG group) improved more than is typical with an HbA1c reduction of $0.6 \%$ at 12 weeks and $0.5 \%$ at 24 weeks. Therefore, at the end of the study, the treatment difference was $0.3 \%$ in favor of the CGM group (Fig. 1a). Similar to the type 1 study, the HbA1c benefit was seen regardless of age, education, or diabetes numeracy. A larger HbAlc reduction was seen in patients with higher baseline HbA1c values, confirming that poor control was not a restricting factor representing inability to adhere to effective CGM utilization. After 24 weeks, ninety-three percent of subjects used the $\mathrm{CGM} \geq 6$ days per week. Seventy-three percent of the patients in the CGM group experienced an $\mathrm{HbA} 1 \mathrm{c}$ reduction of $\geq 0.5 \%$, compared with $49 \%$ in the SMBG group (Fig. 1b). In this study, the time spent in the target range was higher with CGM and the time spent in hyperglycemia was lower in the CGM group, but the frequency of hypoglycemia was very low at baseline in the CGM group (11 min/day). Although it decreased to 4 min per day by the end of the study, the frequency was so low that the decrease relative to no change in the SMBG group was not statistically significant (Fig. 2). This very low frequency of hypoglycemia combined with the decreased $\mathrm{HbA} 1 \mathrm{c}$ benefit in this study, compared with the type 1 study, would suggest the clinical researchers were very conservative in treating these type 2 patients with insulin. Alternatively, the patients themselves may be more conservative in their approach than the type 1 patients. The reason for a conservative approach by clinicians is uncertain, but could relate to a tendency to avoid an aggressive approach in these patients who were much older than the type 1 cohort (60 year mean age vs. 48 year mean). For the patients the limited dose increases and associated lower glucose responses could relate to fear of hypoglycemia or concerns of weight gain. Nevertheless, it appears that CGM was beneficial in most patients with type 2 diabetes treated with CGM and further experience in this population, including customized patient education is likely to increase the benefit seen. Subjects with type 2 diabetes have demonstrated a willingness and ability to use CGM appropriately even when they have been in poor control.

\section{Conclusion}

Over the last decade CGM has become an increasingly refined and valuable tool for real-time monitoring of blood glucose in a variety of diabetes treatment settings and applications. As the accuracy, convenience, and software support have continued to improve, the clinical evidence base has continued to evolve as well. The obvious early application was in patients with type 1 diabetes on intensive insulin therapy to reduce hypoglycemia and improve glucose control. However, the early studies of efficacy in type 1 diabetes were largely proof of concept with mixed diabetes types, exploratory study designs, and mixed insulin delivery methods. Even as studies became more specific for type 1 diabetes, they often represented a disproportionate number of patients on insulin pump therapy and it was difficult to ascertain the specific value in type 1 patients on MDI, the most common insulin delivery method. Recently, the GOLD Trial and the DIAMOND Trial, two randomized controlled trials, have confirmed the independent value of CGM in guiding intensive insulin therapy for type 1 patients treated with MDI. The evidence is clear and allows for practical translation to everyday practice. Yet, the opportunity for further improvement in results will come from added research and experience as it relates to optimal, customized education, and follow-up.

Not surprisingly, the CGM clinical research in type 2 diabetes lagged behind that for type 1 diabetes, particularly with regard to patients treated with MDI. In this increasing patient population, the DIAMOND Trial is the first randomized control trial to address the benefit. The trial confirms benefit in type 2 diabetes treated with MDI as three quarters of the 
patients had an HbA1c improvement of at least $0.5 \%$. However, the study also raises questions as the type 2 cohort experienced less aggressive treatment than seen in the type 1 cohort despite very low levels of hypoglycemia. It seems highly likely that even greater benefit can be seen with more effective patient selection and patient education as well as increased provider familiarity in the years ahead. Yet, the benefit is now clear and expanded use and insurance coverage is warranted.

\section{Author Disclosure Statement}

Andrew Ahmann reports consulting fees from Dexcom and Novo Nordisk as well as research grant funding from Dexcom, Novo Nordisk, Medtronic, and Lexicon.

Janet B. McGill reports consulting fees from Boehringer Ingelheim, Dexcom, Lilly, Merck, Novo Nordisk, Janssen, and Calibra, as well as research funding grants from Novartis, Lexicon, and Dexcom.

\section{References}

1. The Diabetes Control and Complications Trial Research Group: The effect of intensive treatment of diabetes on the development and progression of long-term complications in insulin-dependent diabetes mellitus. N Engl J Med 1993; 329:977-986.

2. UK Prospective Diabetes Study (UKPDS) Group: Effect of intensive blood-glucose control with metformin on complications in overweight patients with type 2 diabetes (UKPDS 34). Lancet (London, England) 1998;352:854-865.

3. UK Prospective Diabetes Study (UKPDS) Group: Intensive blood-glucose control with sulphonylureas or insulin compared with conventional treatment and risk of complications in patients with type 2 diabetes (UKPDS 33). Lancet (London, England) 1998;352:837-853.

4. American Diabetes Association: Pharmacologic approaches to glycemic treatment. Diabetes Care 2017;40:S64-S74.

5. Garber AJ, Abrahamson MJ, Barzilay JI, et al.: Consensus Statement by the American Association of Clinical Endocrinologists and American College of Endocrinology on the comprehensive type 2 diabetes management algorithm2017 executive summary. Endocr Pract 2017;23:207-238.

6. Miller KM, Foster NC, Beck RW, et al.: Current state of type 1 diabetes treatment in the U.S.: updated data from the T1D Exchange clinic registry. Diabetes Care 2015;38:971-978.

7. Nichols GA, Gandra SR, Chiou CF, et al.: Successes and challenges of insulin therapy for type 2 diabetes in a managed-care setting. Curr Med Res Opin 2010;26:9-15.

8. Lipska KJ, Yao X, Herrin J, et al.: Trends in drug utilization, glycemic control, and rates of severe hypoglycemia, 2006-2013. Diabetes Care 2017;40:468-475.

9. Miller KM, Beck RW, Bergenstal RM, et al.: Evidence of a strong association between frequency of self-monitoring of blood glucose and hemoglobin A1c levels in T1D exchange clinic registry participants. Diabetes Care 2013;36:2009-2014.

10. Gross TM, Bode BW, Einhorn D, et al.: Performance evaluation of the MiniMed continuous glucose monitoring system during patient home use. Diabetes Technol Ther 2000;2:49-56.

11. Rodbard D: Continuous glucose monitoring: a review of successes, challenges, and opportunities. Diabetes Technol Ther 2016;18 Suppl 2:S3-S13.

12. American Diabetes Association: Glycemic Targets. Diabetes Care 2017;40:S48-S56.
13. Fonseca VA, Grunberger G, Anhalt H, et al.: Continuous glucose monitoring: a consensus conference of the American Association of Clinical Endocrinologists and American College of Endocrinology. Endocr Pract 2016;22: 1008-1021.

14. Peters AL, Ahmann AJ, Battelino T, et al.: Diabetes technology-continuous subcutaneous insulin infusion therapy and continuous glucose monitoring in adults: an Endocrine Society Clinical Practice Guideline. J Clin Endocrinol Metab 2016;101:3922-3937.

15. Yeh HC, Brown TT, Maruthur N, et al.: Comparative effectiveness and safety of methods of insulin delivery and glucose monitoring for diabetes mellitus: a systematic review and meta-analysis. Ann Intern Med 2012;157:336-347.

16. Simmons JH, Chen V, Miller KM, et al.: Differences in the management of type 1 diabetes among adults under excellent control compared with those under poor control in the T1D Exchange Clinic Registry. Diabetes Care 2013;36: 3573-3577.

17. Orchard TJ, Nathan DM, Zinman B, et al.: Association between 7 years of intensive treatment of type 1 diabetes and long-term mortality. JAMA 2015;313:45-53.

18. Lind M, Svensson AM, Kosiborod M, et al.: Glycemic control and excess mortality in type 1 diabetes. $\mathrm{N}$ Engl $\mathrm{J}$ Med 2014;371:1972-1982.

19. Writing Group for the DCCT/EDIC Research Group. Mortality in type 1 diabetes in the DCCT/EDIC versus the general population. Diabetes Care 2016;39:1378-1383.

20. Writing Group for the DCCT/EDIC Research Group. Intensive diabetes treatment and cardiovascular outcomes in type 1 diabetes: the DCCT/EDIC study 30-year followup. Diabetes Care 2016;39:686-693.

21. Gagnum V, Stene LC, Leivestad T, et al.: Long-term mortality and end-stage renal disease in a type 1 diabetes population diagnosed at age 15-29 years in Norway. Diabetes Care 2017;40:38-45.

22. Livingstone SJ, Looker HC, Hothersall EJ, et al.: Risk of cardiovascular disease and total mortality in adults with type 1 diabetes: Scottish registry linkage study. PLoS Med 2012;9:e1001321.

23. Tu E, Twigg SM, Duflou J, Semsarian C: Causes of death in young Australians with type 1 diabetes: a review of coronial postmortem examinations. Med J Aust 2008;188: 699-702.

24. Gagnum V, Stene LC, Jenssen TG, et al.: Causes of death in childhood-onset Type 1 diabetes: long-term follow-up. Diabet Med 2017;34:56-63.

25. Frier BM: Hypoglycaemia in diabetes mellitus: epidemiology and clinical implications. Nat Rev Endocrinol 2014;10:711-722.

26. Tanenberg RJ, Newton CA, Drake AJ: Confirmation of hypoglycemia in the "dead-in-bed" syndrome, as captured by a retrospective continuous glucose monitoring system. Endocr Pract 2010;16:244-248.

27. Novodvorsky P, Bernjak A, Chow E, et al.: Diurnal differences in risk of cardiac arrhythmias during spontaneous hypoglycemia in young people with type 1 diabetes. Diabetes Care 2017;40:655-662.

28. UK Hypoglycaemia Study Group: Risk of hypoglycaemia in types 1 and 2 diabetes: effects of treatment modalities and their duration. Diabetologia 2007;50:1140-1147.

29. Thomas RM, Aldibbiat A, Griffin W, et al.: A randomized pilot study in Type 1 diabetes complicated by severe hypoglycaemia, comparing rigorous hypoglycaemia avoidance 
with insulin analogue therapy, CSII or education alone. Diabet Med 2007;24:778-783.

30. Pickup JC, Sutton AJ: Severe hypoglycaemia and glycaemic control in Type 1 diabetes: meta-analysis of multiple daily insulin injections compared with continuous subcutaneous insulin infusion. Diabet Med 2008;25:765-774.

31. Garg SK, Schwartz S, Edelman SV: Improved glucose excursions using an implantable real-time continuous glucose sensor in adults with type 1 diabetes. Diabetes Care 2004;27:734-738.

32. Bailey TS, Zisser HC, Garg SK: Reduction in hemoglobin A1C with real-time continuous glucose monitoring: results from a 12-week observational study. Diabetes Technol Ther 2007;9:203-210.

33. Tamborlane WV, Beck RW, Bode BW, et al.: Continuous glucose monitoring and intensive treatment of type 1 diabetes. N Engl J Med 2008;359:1464-1476.

34. Bode B, Beck RW, Xing D, et al.: Sustained benefit of continuous glucose monitoring on A1C, glucose profiles, and hypoglycemia in adults with type 1 diabetes. Diabetes Care 2009;32:2047-2049.

35. Mauras N, Beck R, Xing D, et al.: A randomized clinical trial to assess the efficacy and safety of real-time continuous glucose monitoring in the management of type 1 diabetes in young children aged 4 to $<10$ years. Diabetes Care 2012;35:204-210.

36. Riveline JP, Schaepelynck P, Chaillous L, et al.: Assessment of patient-led or physician-driven continuous glucose monitoring in patients with poorly controlled type 1 diabetes using basal-bolus insulin regimens: a 1-year multicenter study. Diabetes Care 2012;35:965-971.

37. Garg SK, Beatson CR, Miller HA, et al.: Use of continuous glucose monitoring in subjects with type 1 diabetes on multiple daily injections versus continuous subcutaneous insulin infusion therapy. Diabetes Care 2011;34:574-579.

38. Little SA, Leelarathna L, Walkinshaw E, et al.: Recovery of hypoglycemia awareness in long-standing type 1 diabetes: a multicenter $2 \times 2$ factorial randomized controlled trial comparing insulin pump with multiple daily injections and continuous with conventional glucose self-monitoring (HypoCOMPaSS). Diabetes Care 2014;37:2114-2122.

39. van Beers CA, DeVries JH, Kleijer SJ, et al.: Continuous glucose monitoring for patients with type 1 diabetes and impaired awareness of hypoglycaemia (IN CONTROL): a randomised, open-label, crossover trial. Lancet Diabetes Endocrinol 2016;4:893-902.

40. Battelino T, Phillip M, Bratina N, et al.: Effect of continuous glucose monitoring on hypoglycemia in type 1 diabetes. Diabetes Care 2011;34:795-800.

41. Foster NC, Miller KM, Tamborlane WV, et al.: Continuous glucose monitoring in patients with type 1 diabetes using insulin injections. Diabetes Care 2016;39:e81-e82.

42. Beck RW, Riddlesworth T, Ruedy K, et al.: Effect of continuous glucose monitoring on glycemic control in adults with type 1 diabetes using insulin injections: the DIAMOND Randomized Clinical Trial. JAMA 2017;317:371-378.

43. Lind M, Polonsky W, Hirsch IB, et al.: Continuous glucose monitoring vs conventional therapy for glycemic control in adults with type 1 diabetes treated with multiple daily insulin injections: the GOLD Randomized Clinical Trial. JAMA 2017;317:379-387.

44. Davidson MB: Continuous glucose monitoring in patients with type 1 diabetes taking insulin injections. JAMA 2017; $317: 363-364$.
45. Lawrence JM, Imperatore G, Dabelea D, et al.: Trends in incidence of type 1 diabetes among non-Hispanic white youth in the U.S., 2002-2009. Diabetes 2014;63:39383945.

46. Bronstone A, Graham C: The potential cost implications of averting severe hypoglycemic events requiring hospitalization in high-risk adults with type 1 diabetes using realtime continuous glucose monitoring. J Diabetes Sci Technol 2016;10:905-913.

47. Allen NA, Fain JA, Braun B, Chipkin SR: Continuous glucose monitoring counseling improves physical activity behaviors of individuals with type 2 diabetes: a randomized clinical trial. Diabetes Res Clin Pract 2008;80:371-379.

48. Yoo HJ, An HG, Park SY, et al.: Use of a real time continuous glucose monitoring system as a motivational device for poorly controlled type 2 diabetes. Diabetes Res Clin Pract 2008;82:73-79.

49. Bergenstal RM, Rosenstock J, Bastyr EJ, 3rd, et al.: Lower glucose variability and hypoglycemia measured by continuous glucose monitoring with novel long-acting insulin LY2605541 versus insulin glargine. Diabetes Care 2014;37: 659-665.

50. Nagakura J, Yamakawa T, Taguri M, et al.: Effects of exenatide and liraglutide on 24-hour glucose fluctuations in type 2 diabetes. Endocr J 2016;63:239-247.

51. van Dijk P, Bouma A, Landman GW, et al.: Hypoglycemia in frail elderly patients with type 2 diabetes mellitus treated with sulfonylurea: the results of continuous glucose monitoring. J Diabetes Sci Technol 2016;11:438-439.

52. Investigators F-ST: Glucose variability in a 26-week randomized comparison of mealtime treatment with rapidacting insulin versus GLP-1 agonist in participants with type 2 diabetes at high cardiovascular risk. Diabetes Care 2016;39:973-981.

53. Holst JJ, Buse JB, Rodbard HW, et al.: IDegLira improves both fasting and postprandial glucose control as demonstrated using continuous glucose monitoring and a standardized meal test. J Diabetes Sci Technol 2015;10:389-397.

54. Li FF, Jiang LL, Yan RN, et al.: Effects of saxagliptin addon therapy to insulin on blood glycemic fluctuations in patients with type 2 diabetes: a randomized, control, openlabeled trial. Medicine 2016;95:e5229.

55. Wang JS, Lee IT, Lee WJ, et al.: Glycemic excursions were positively associated with $\mathrm{HbA1c}$ reduction from baseline after treatment with acarbose in patients with type 2 diabetes on metformin monotherapy. J Diabetes 2017;9:248-255.

56. Vigersky R, Shrivastav M: Role of continuous glucose monitoring for type 2 in diabetes management and research. J Diabetes Complications 2017;31:280-287.

57. Cariou B, Fontaine P, Eschwege E, et al.: Frequency and predictors of confirmed hypoglycaemia in type 1 and insulin-treated type 2 diabetes mellitus patients in a real-life setting: results from the DIALOG study. Diabetes Metab 2015;41:116-125.

58. Gehlaut RR, Dogbey GY, Schwartz FL, et al.: Hypoglycemia in type 2 diabetes-More Common Than You Think: a Continuous Glucose Monitoring Study. J Diabetes Sci Technol 2015;9:999-1005.

59. Lipska KJ, Warton EM, Huang ES, et al.: HbA1c and risk of severe hypoglycemia in type 2 diabetes: the Diabetes and Aging Study. Diabetes Care 2013;36:3535-3542.

60. Pazos-Couselo M, Garcia-Lopez JM, Gonzalez-Rodriguez M, et al.: High incidence of hypoglycemia in stable insulintreated type 2 diabetes mellitus: continuous glucose 
monitoring vs. self-monitored blood glucose. Observational prospective study. Can J Diabetes 2015;39:428-433.

61. Wright AD, Cull CA, Macleod KM, Holman RR: Hypoglycemia in Type 2 diabetic patients randomized to and maintained on monotherapy with diet, sulfonylurea, metformin, or insulin for 6 years from diagnosis: UKPDS73. J Diabetes Complications 2006;20:395-401.

62. Zoungas S, Patel A, Chalmers J, et al.: Severe hypoglycemia and risks of vascular events and death. N Engl J Med 2010;363:1410-1418.

63. Hanefeld M, Duetting E, Bramlage P: Cardiac implications of hypoglycaemia in patients with diabetes - a systematic review. Cardiovasc Diabetol 2013;12:135.

64. Lee AS, Brooks BA, Simmons L, et al.: Hypoglycaemia and QT interval prolongation: detection by simultaneous Holter and continuous glucose monitoring. Diabetes Res Clin Pract 2016;113:211-214.

65. Marques JL, George E, Peacey SR, et al.: Altered ventricular repolarization during hypoglycaemia in patients with diabetes. Diabet Med 1997;14:648-654.

66. Scheiner G: CGM retrospective data analysis. Diabetes Technol Ther 2016;18 Suppl 2:S214-S222.

67. Haviland N, Walsh J, Roberts R, Bailey TS: Update on clinical utility of continuous glucose monitoring in type 1 diabetes. Curr Diabetes Rep 2016;16:115.

68. Ehrhardt NM, Chellappa M, Walker MS, et al.: The effect of real-time continuous glucose monitoring on glycemic control in patients with type 2 diabetes mellitus. J Diabetes Sci Technol 2011;5:668-675.

69. Fonda SJ, Salkind SJ, Walker MS, et al.: Heterogeneity of responses to real-time continuous glucose monitoring (RTCGM) in patients with type 2 diabetes and its implications for application. Diabetes Care 2013;36:786-792.

70. Vigersky RA, Fonda SJ, Chellappa M, et al.: Short- and longterm effects of real-time continuous glucose monitoring in patients with type 2 diabetes. Diabetes Care 2012;35:32-38.

71. Garg S, Zisser H, Schwartz S, et al.: Improvement in glycemic excursions with a transcutaneous, real-time continu- ous glucose sensor: a randomized controlled trial. Diabetes Care 2006;29:44-50.

72. Cosson E, Hamo-Tchatchouang E, Dufaitre-Patouraux L, et al.: Multicentre, randomised, controlled study of the impact of continuous subcutaneous glucose monitoring (GlucoDay) on glycaemic control in type 1 and type 2 diabetes patients. Diabetes Metab 2009;35:312-318.

73. New JP, Ajjan R, Pfeiffer AF, Freckmann G: Continuous glucose monitoring in people with diabetes: the randomized controlled Glucose Level Awareness in Diabetes Study (GLADIS). Diabet Med 2015;32:609-617.

74. Murphy HR, Rayman G, Lewis K, et al.: Effectiveness of continuous glucose monitoring in pregnant women with diabetes: randomised clinical trial. BMJ 2008;337:a1680.

75. Kepenekian L, Smagala A, Meyer L, et al.: Continuous glucose monitoring in hemodialyzed patients with type 2 diabetes: a multicenter pilot study. Clin Nephrol 2014;82: 240-246.

76. Haak T, Hanaire H, Ajjan R, et al.: Flash glucose-sensing technology as a replacement for blood glucose monitoring for the management of insulin-treated type 2 diabetes: a multicenter, open-label randomized controlled trial. Diabetes Ther 2017;8:55-73.

77. Bergenstal RRT, Ruedy K, Kolman C, et al.: Patients with type 2 diabetes using multiple daily insulin injections have high adherence and benefit from CGM: a prospective, randomized controlled trial. Diabetes Technol Ther 2017; 19(Suppl 1):A11 (ATTD abstract 30).

Address correspondence to: Andrew Ahmann, MD Oregon Health \& Science University 3181 SW Sam Jackson Park Road, OP05DC Portland, OR 97239

E-mail: ahmanna@ohsu.edu 